\title{
A NEW CHARACTERIZATION OF DEDEKIND DOMAINS
}

\author{
by D. D. ANDERSON and E. W. JOHNSON
}

(Received 23 September, 1985)

Throughout this paper all rings are assumed commutative with identity. Among integral domains, Dedekind domains are characterized by the property that every ideal is a product of prime ideals. For a history and proof of this result the reader is referred to Cohen [2, pp. 31-32]. More generally, Mori [5] has shown that a ring has the property that every ideal is a product of prime ideals if and only if it is a finite direct product of Dedekind domains and special principal ideal rings (SPIRS). Rings with this property are called general Z.P.I.-rings.

Since in a Dedekind domain every nonzero ideal is a finite intersection of powers of maximal ideals, it follows that in a general Z.P.I.-ring every ideal is a finite intersection of powers of prime ideals. Butts and Gilmer [1] proved the converse.

The purpose of this paper is to generalize and unify the results of Mori and Butts and Gilmer. Hence, if $R$ is a ring, we denote by $\mathscr{L}(R)$ the set of ideals of $R$, by $\mathscr{P}(R)$ the set of prime ideals of $R$ and by $\mathscr{P}(R)$ the closure of $\mathscr{P}(R)$ under products and finite intersections. For convenience we set $R=P^{0}$ for any prime ideal $P$, so that $R \in \mathscr{P}(R)$. We show that $R$ is a general Z.P.I.-ring if and only if $\mathscr{L}(R)=\mathscr{P}(R)$.

We note three elementary but useful facts. If $A \in \mathscr{P}(R), A \neq R$, then there are only finitely many prime ideals minimal over $A$. If $A \in \mathscr{P}(R)$ and $S$ is a multiplicatively closed subset of $R$, then $A_{s} \in \mathscr{P}\left(R_{S}\right)$. And if $A$ and $B$ are ideals with $A \subseteq B$ and $B \in \mathscr{P}(R)$, then $B / A \in \mathscr{P}(R / A)$. In particular, the property $\mathscr{L}(R)=\mathscr{P}(R)$ carries over to $R_{S}$ and to $R / A$.

LEMMA 1 . Let $R$ be an integral domain satisfying $\mathscr{L}(R)=\mathscr{P}(R)$. Let $P$ be a prime ideal of $R$ minimal over a nonzero principal ideal. Then $R_{P}$ is a DVR and hence rank $P=1$.

Proof. Let $P$ be minimal over $(a)$. Then $R_{P}$ satisfies $\mathscr{L}\left(R_{P}\right)=\mathscr{P}\left(R_{P}\right)$ and $P_{P}$ is minimal over $(a)_{P}$. Thus we can assume that $(R, M)$ is a quasilocal domain satisfying $\mathscr{L}(R)=\mathscr{P}(R)$ and that $M$ is minimal over $(a)$. We must show that $R$ is a discrete valuation ring (DVR). Since $M$ is the only prime ideal containing (a), $(a)$ must be a power of $M$. Hence $M$ is invertible. Since $R$ is quasilocal, $M$ is principal, say $M=(p)$. If $\bigcap_{n=1}^{\infty}\left(p^{n}\right)=0$, then $(p)$ is the only nonzero prime ideal of $R$, so $R$ is a DVR. Hence we may assume that $0 \neq b \in \bigcap_{n=1}^{\infty}\left(p^{n}\right)$. Since each prime $Q \subsetneq(p)$ satisfies $Q=p Q$ and since for $A$ and $B$ with $p A=A$ and $p B=B$, we have $p(A B)=A B$ and $p(A \cap B)=p A \cap p B=A \cap B$, it follows from $(b) \in \mathscr{P}(R)$ that $(b)=p(b)$. Thus by Nakayama's Lemma, $(b)=0$. Thus $R$ must be a DVR.

LEMma 2. Let $(R, M)$ be a quasilocal ring satisfying $\mathscr{L}(R)=\hat{\mathscr{P}}(R)$. Then either $M=M^{2}$ or $M$ is principal.

Glasgow Math. J. 28 (1986) 237-239. 
Proof. Suppose that $M \neq M^{2}$. Let $x \in M-M^{2}$. Let $y \in M$, then since $\left(x, y^{2}\right) \in \mathscr{P}$ and $\left(x, y^{2}\right) \notin M^{2},\left(x, y^{2}\right)$ must be an intersection of prime ideals. Hence $(x, y) \subseteq \sqrt{ }\left(x, y^{2}\right)$, so $(x, y)=\left(x, y^{2}\right)$. By Nakayama's Lemma, $(x, y)=(x)$. Since $y$ was arbitrary, $M=(x)$.

THEOREM 3. Let $R$ be an integral domain. Then $R$ satisfies $\mathscr{L}(R)=\mathscr{P}(R)$ if and only if $R$ is a Dedekind domain.

Proof. Since every ideal in a Dedekind domain is a product of prime ideals, a Dedekind domain satisfies $\mathscr{L}(R)=\mathscr{P}(R)$. Conversely, suppose that $\mathscr{L}(R)=\mathscr{P}(R)$ and that $R$ is not a field. It suffices to show that every rank one prime ideal of $R$ is maximal. For then by Lemma $1, R_{M}$ will be a DVR for each maximal ideal $M$ of $R$. Also, for $0 \neq b \in R,(b) \in \mathscr{P}(R)$ and hence $\sqrt{ }(b)$ is a finite intersection of prime ideals, so $(b)$ is contained in only finitely many maximal ideals of $R$. Then by [3, Theorem 37.2], $R$ will be a Dedekind domain. Let $P$ be a rank one prime ideal of $R$. Assume that $P$ is not maximal, say $P \subsetneq M$, a maximal ideal. Let $0 \neq a \in P$. Let the other rank one prime ideals containing (a) be $P_{1}, \ldots, P_{n}$. Choose $b \in M-\left(P \cup P_{1} \cup \ldots \cup P_{n}\right)$. Hence $(a, b) \subseteq M$, but $(a, b)$ is contained in no rank one prime ideal. Shrink $M$ to a prime ideal $Q$ minimal over $(a, b)$. Pass to $R_{Q}$. Then $\mathscr{L}\left(R_{Q}\right)=\mathscr{P}\left(R_{Q}\right)$ and $Q_{Q}$ is minimal over $(a, b)_{Q}$, so $(a, b)_{Q}=Q_{Q}^{s}$ for some $s \geqslant 1$. Hence by Nakayama's Lemma, we must have $Q_{Q} \neq Q_{Q}^{2}$. By Lemma $2, Q_{Q}$ is principal. But then by Lemma $1, R_{Q}$ is a DVR, so rank $Q=1$. This contradiction shows that $P$ must be maximal.

It may be of interest to note that to this point we have used only that for all $x, y \in R$ (an integral domain), $(x) \in \mathscr{P}(R),(y) \in \mathscr{P}(R)$ and $(x)+(y) \in \mathscr{P}(R)$.

THEOREM 4. A ring $R$ has the property that every proper ideal is in $\mathscr{P}(R)$ if and only if $R$ is a general Z.P.I.-ring.

Proof. If $R$ is a general Z.P.I.-ring, then every proper ideal is a product of prime ideals and hence is in $\mathscr{P}(R)$. Conversely, suppose that every proper ideal of $R$ is in $\mathscr{P}(R)$. If $P$ is a prime ideal of $R$, then $\mathscr{L}(R / P)=\mathscr{P}(R / P)$ and hence by Theorem $3 R / P$ is a Dedekind domain. It follows that $\operatorname{dim} R \leqslant 1$. Let $M$ be a maximal ideal of $R$. We show that $R_{M}$ is either a SPIR or a DVR. Then since (0) is a product of prime ideals of $R$ and since for each maximal ideal $M$ of $R, R_{M}$ is a SPIR or a DVR, it easily follows that $R$ is a finite direct product of SPIRs and Dedekind domains. (See, for example, the proof of $[3$, Theorem 46.11].) Thus we are reduced to proving that a quasilocal ring $(R, M)$ with $\operatorname{dim} R \leqslant 1$ satisfying $\mathscr{L}(R)=\mathscr{P}(R)$ is either a SPIR or a DVR. If $\operatorname{dim} R=0$, then every principal ideal of $R$ is a power of $M$ and hence $R$ is a SPIR. Suppose that $\operatorname{dim} R=1$. Since $R$ has only finitely many minimal prime ideals, $M$ is minimal over a principal ideal $(a)$. Thus $(a)$ is a power of $M$, so $M \neq M^{2}$. Hence by Lemma $2, M$ is principal. Hence $P=\bigcap_{n=1}^{\infty} M^{n}=M P$ is the unique minimal prime ideal of $R$. Since every principal ideal of $R$ is a power of $M$ or of $P$ it easily follows that $P$ is principal. Hence $P=0$. So $R$ is a DVR.

Along the lines of the note following Theorem 3, we note here that Theorem 4 remains 
valid under the assumption that every element of $\mathscr{L}(R)$ is the sum (not necessarily finite) of elements of $\mathscr{P}(R)$ and that $A, B \in \mathscr{P}(R)$ implies $A+B \in \hat{\mathscr{P}}(R)$.

ACKNOwLEDgement. The authors wish to acknowledge the support services provided at University House, The University of Iowa.

\section{REFERENCES}

1. H. S. Butts and R. W. Gilmer, Primary ideals and prime power ideals, Canad. J. Math. 18 (1966), 1183-1195.

2. I. S. Cohen, Commutative rings with restricted minimum condition, Duke Math. J 17 (1950), 27-42.

3. R. W. Gilmer, Multiplicative Ideal Theory (Marcel Dekker, 1972).

4. K. B. Levitz, A characterization of general Z.P.I.-rings II, Pacific J. Math. 42 (1972), 147-151.

5. S. Mori, Allgemeine Z.P.I.-ringe, J. Sci. Hirosima Univ. Ser. A. 10 (1940), 117-136.

UNIVERSITY OF IOWA

IOWA CITY

IowA 52242, USA 